\title{
TOWARDS THE FIXED POINT PROPERTY FOR SUPERREFLEXIVE SPACES
}

\author{
ANDRZEJ WIŚNICKI
}

\begin{abstract}
A Banach space $X$ is said to have property $\left(S_{m}\right)$ if every metrically convex set $A \subset X$ which lies on the unit sphere and has diameter not greater than one can be (weakly) separated from zero by a functional. We show that this geometrical condition is closely connected with the fixed point property for nonexpansive mappings in superreflexive spaces.
\end{abstract}

\section{INTRODUCTION}

Let $C$ be a nonempty, bounded, closed and convex subset of a Banach space $X$ and let $T: C \rightarrow C$ be a nonexpansive mapping, that is, $\|T x-T y\| \leqslant\|x-y\|$ for all $x, y \in C$. We say that $X$ has the fixed point property (FPP in short) if every such mapping has a fixed point.

Fixed point theory for nonexpansive mappings has its origins in 1965, when Browder [3] proved that a Hilbert space has FPP. In the same year Browder [4] and Göhde [11] showed that all uniformly convex spaces have FPP and Kirk [16] proved more general result stating that all Banach spaces with the so-called normal structure have the fixed point property for weakly compact, convex sets. In particular, all reflexive spaces with normal structure have FPP. The problem whether reflexivity implies the fixed point property and the converse question, in spite of many investigations in this direction, are both still open.

However, there are some partial results concerning this problem. In [18] Maurey used the Banach space ultraproduct construction to prove the fixed point property for all reflexive subspaces of $L_{1}[0,1]$. He also showed that isometries in superreflexive spaces always have FPP. Note that quite recently Dowling and Lennard [6] have proved that every nonreflexive subspace of $L_{1}[0,1]$ fails FPP.

The ultrapower techniques of Maurey have been extended by many authors and a lot of strong and deep results in metric fixed point theory have been obtained in this way (see for instance $[1,7,9,17,19,20]$ ).

Received 22nd March, 2001

The research was supported in part by KBN grant 2 PO3A 02915.

Copyright Clearance Centre, Inc. Serial-fee code: 0004-9727/01 \$A2.00+0.00. 
In the present paper we use these techniques together with other "nonstandard" facts about Banach spaces to propose a new approach to FPP problem in the case of superreflexive spaces. We start with the following

DEFinition 1.1. We say that a Banach space $X$ has property $(S)$ if for every subset $A$ of the unit sphere $S_{X}$ with $\operatorname{diam} A \leqslant 1$ there exists a functional $F$ in the dual space $X^{*}$ such that $F(x)>0$ for all $x \in A$.

In Section 4 we show that all separable spaces as well as all strictly convex spaces have the $(S)$ property. On the other hand, there exist superreflexive spaces, which do not possess that property.

For our purpose we shall need a slightly weaker property. Recall that a closed set $A$ is said to be metrically convex if for every $x, y \in A$ there exists $z \in A$ such that $\|x-z\|=\|x-y\| / 2$ and $\|y-z\|=\|x-y\| / 2$. Note that in the case of strictly convex spaces this notion is equivalent to convexity.

Definition 1.2. We say that a Banach space $X$ has property $\left(S_{m}\right)$ if for every metrically convex set $A \subset S_{X}$ with $\operatorname{diam} A \leqslant 1$ there exists $F \in X^{*}$ such that $F(x)>0$ for all $x \in A$.

We point out that the related property obtained by replacing the words "metrically convex" by "convex" is satisfied by every Banach space (by the Hahn-Banach Theorem). It is not known whether there exists a Banach space without property $\left(S_{m}\right)$.

The following two open questions are of central importance to our paper:

1. Does every superreflexive space have property $\left(S_{m}\right)$ ?

2. Does every space isomorphic to a Hilbert space have property $\left(S_{m}\right)$ ?

It will follow from Theorem 3.1 in Section 3 that positive answers to these questions yield positive solutions to FPP problem in these cases.

Further applications of Theorem 3.1 in fixed point theory are given in Section 4. In particular, we show that all uniformly noncreasy spaces, a large class of superreflexive spaces introduced by Prus in [19], have property $\left(S_{m}\right)$.

\section{Preliminaries}

We shall briefly recall the construction of Banach ultrapowers. For more details we refer the reader to $[\mathbf{1}, \mathbf{1 0}, \mathbf{2 0}]$. Let $U$ be a countably incomplete ultrafilter defined on a set $I$. The ultrapower $(X)_{U}$ of a Banach space $X$ is the quotient space of

$$
l_{\infty}(X)=\left\{\left(x_{n}\right): x_{n} \in X \text { for all } n \in I \text { and }\left\|\left(x_{n}\right)\right\|=\sup _{n}\left\|x_{n}\right\|<\infty\right\}
$$

by $\operatorname{ker} \mathcal{N}=\left\{\left(x_{n}\right) \in l_{\infty}(X): \lim _{U}\left\|x_{n}\right\|=0\right\}$. Here $\lim _{U}$ denotes the ultralimit over $U$. One can prove that the quotient norm on $(X)_{U}$ is given by $\left\|\left(x_{n}\right)_{U}\right\|=\lim _{U}\left\|x_{n}\right\|$, where $\left(x_{n}\right)_{U}$ is the equivalence class of $\left(x_{n}\right)$. It is also clear that $X$ is isometric to a subspace 
of $(X)_{U}$ by the mapping $x \rightarrow(x)_{U}$. Therefore, we shall assume that $X$ is a subspace of $(X)_{U}$

Let us now recall two characterisations of superreflexive spaces.

TheOREM 2.1. (James [15] and Enflo [8].) For a Banach space $X$ the following are equivalent:

1. $X$ is superreflexive.

2. $X$ has an equivalent uniformly convex norm.

3. $X$ has an equivalent uniformly nonsquare norm.

ThEOREM 2.2 (Henson-Moore $[\mathbf{1 3}, \mathbf{1 4}]$. . Let $U$ be a countably incomplete ultrafilter on a set $I$. The following conditions are equivalent:

1. $X$ is superreflexive.

2. $(X)_{U}$ is reflexive.

3. $(X)_{U}$ is superreflexive.

4. $\left(X^{*}\right)_{U}$ is isometric onto $\left((X)_{U}\right)^{*}$ by the canonical isometric isomorphism.

We shall heavily use the last statement in the next section so let us give some explanations. The canonical isometric isomorphism is defined as

$$
(J f)(x)=\lim _{U} f_{n}\left(x_{n}\right),
$$

where $f=\left(f_{n}\right)_{U} \in\left(X^{*}\right)_{U}$ and $x=\left(x_{n}\right)_{U} \in(X)_{U}$. It is not very difficult to prove that $J$ is a well defined linear operator and $\|J f\|=\|f\|$. In general $J$ is only an isometric embedding of $\left(X^{*}\right)_{U}$ into $\left((X)_{U}\right)^{*}$. Theorem 2.2 states that in the case of superreflexive spaces we may identify the ultrapower $\left(X^{*}\right)_{U}$ with the dual $\left((X)_{U}\right)^{*}$. Thus, in this case, each element $F \in\left((X)_{U}\right)^{*}$ is represented by $\left(f_{n}\right)_{U} \in\left(X^{*}\right)_{U}$ (see $[\mathbf{1 2}, \mathbf{2 0}$ ] for more details).

\section{Main Result}

In this section we shall prove our main Theorem 3.1. To make the proof self-contained we shall briefly recall some facts from metric fixed point theory.

Let $C$ be a nonempty weakly compact, convex subset of a Banach space $X$ and assume that there exists a nonexpansive mapping $T: C \rightarrow C$ without fixed points. Then, by Zorn's Lemma, there exists a minimal (in the sense of inclusion) convex and weakly compact set $K \subset C$ which is invariant under $T$ and which is not a singleton. Let us recall basic properties of such sets:

(a) $\overline{c o n v} T(K)=K$,

(b) $K$ is diametral, that is, $\sup _{y \in K}\|x-y\|=\operatorname{diam} K$ for all $x \in K$, 
(c) if $\left(x_{n}\right) \subset K$ is an approximate fixed point sequence (that is, $\lim _{n \rightarrow \infty} \| T x_{n}-$ $x_{n} \|=0$ ), then $\lim _{n \rightarrow \infty}\left\|x_{n}-x\right\|=\operatorname{diam} K$ for all $x \in K$.

The last property is known as the Goebel-Karlovitz Lemma. We shall also need a certain generalisation of this result:

$\left(\mathbf{c}^{\prime}\right) \quad$ if $\left(x_{n}\right) \subset K$ satisfies the condition $\lim _{U}\left\|T x_{n}-x_{n}\right\|=0$, then $\lim _{U}\left\|x_{n}-x\right\|=$ $\operatorname{diam} K$ for all $x \in K$.

To translate these properties into ultraproduct language, take a free ultrafilter on $\mathbb{N}$ and consider the set $\widetilde{K}=(K)_{U} \subset(X)_{U}$ defined by

$$
\widetilde{K}=\left\{\left(x_{n}\right)_{U} \in(X)_{U}: x_{n} \in K \text { for all } n \in \mathbb{N}\right\}
$$

and the mapping $\widetilde{T}: \widetilde{K} \rightarrow \widetilde{K}$ by setting $\widetilde{T}\left(\left(x_{n}\right)_{U}\right)=\left(T x_{n}\right)_{U}$. It is easy to see that $\operatorname{Fix} \tilde{T}$, the set of fixed points of $\widetilde{T}$, is nonempty and is characterised as those points from $\widetilde{K}$ represented by sequences $\left(x_{n}\right)$ in $K$ for which $\lim _{U}\left\|T x_{n}-x_{n}\right\|=0$. Using (c'), we obtain the following properties:

(i) $\operatorname{diam} \operatorname{Fix} \widetilde{T}=\operatorname{diam} K=\operatorname{diam} \tilde{K}$,

(ii) $K, \widetilde{K}$, Fix $\widetilde{T}$ are diametral,

(iii) $\|x-\tilde{y}\|=\operatorname{diam} K$ for every $x \in K$ and $\tilde{y} \in \operatorname{Fix} \tilde{T}$.

Moreover, it was proved by Maurey $[\mathbf{1 8}]$ (see also $[\mathbf{1}, \mathbf{2 0}]$ ) that

(iv) Fix $\widetilde{T}$ is metrically convex.

We can now use the whole machinery described above to prove

THEOREM 3.1. Let $X$ be a superreflexive space and assume that there exists a free ultrafilter $U$ on $\mathbb{N}$ such that $(X)_{U}$ has property $\left(S_{m}\right)$. Then $X$ has FPP.

ProOF: Assume conversely that there exists a minimal, closed, convex and bounded set $K$ and a nonexpansive mapping $T: K \rightarrow K$ without fixed points. Let $\left(x_{n}\right)$ be an approximate fixed point sequence. There is no loss of generality in assuming that $\left(x_{n}\right)$ tends weakly to $0 \in K$ and $\operatorname{diam} K=1$. Hence, Fix $\tilde{T}$ is a metrically convex subset of the unit sphere and has diameter 1.

Since $(X)_{U}$ has property $\left(S_{m}\right)$, there exists $F \in\left((X)_{U}\right)^{*}$ such that $F(x)>0$ for all $x \in$ Fix $\tilde{T}$. By Theorem $2.2, F$ is represented by an element $\left(f_{n}\right)_{U} \in\left(X^{*}\right)_{U}$. Since $\left(x_{n}\right)$ tends weakly to zero, we can obtain a subsequence $\left(x_{n_{k}}\right)$ of $\left(x_{n}\right)$ such that $\left|f_{k}\left(x_{n_{k}}\right)\right|<1 / k$ for all $k \in \mathbb{N}$. But $\left(x_{n}\right)$ is an approximate fixed point sequence so $\lim _{k \rightarrow \infty} \| T x_{n_{k}}-$ $x_{n_{k}} \|=0$ and consequently $\left(x_{n_{k}}\right)_{U} \in \operatorname{Fix} \tilde{T}$. Hence $F\left(\left(x_{n_{k}}\right)_{U}\right)>0$. On the other hand. $F\left(\left(x_{n_{k}}\right)_{U}\right)=\lim _{U} f_{k}\left(x_{n_{k}}\right)=0$ and we obtain a contradiction.

Since Banach ultrapowers preserve superreflexivity as well as the isomorphism to a Hilbert space we obtain the following consequences of Theorem 3.1.

CoRollary 3.2. If all superreflexive spaces have property $\left(S_{m}\right)$, then all superreflexive spaces have FPP. 
COROLLARY 3.3. If all spaces isomorphic to a Hilbert space have property $\left(S_{m}\right)$, then all such spaces have FPP.

\section{Applications}

We shall first study Banach spaces with property $(S)$. Recall that $\varepsilon_{0}(X)=\sup \{\varepsilon \geqslant$ $\left.0: \delta_{X}(\varepsilon)=0\right\}$, where $\delta_{X}$ is the modulus of convexity of a Banach space $X$. It is well known that the condition $\varepsilon_{0}(X)<2$ implies superreflexivity of $X$.

Proposition 4.1. The following classes of Banach spaces have property $(S)$ :

(i) all separable Banach spaces,

(ii) all strictly convex spaces,

(iii) all Banach spaces $X$ with $\varepsilon_{0}(X)<1$.

Proof: (i) Let $A$ be a subset of the unit sphere $S_{X}$ with $\operatorname{diam} A \leqslant 1$. Assume first that $A$ is countable. For each $x_{i} \in A, i=1,2, \ldots$, take a supporting functional $f_{i} \in S_{X}$. with $f_{i}\left(x_{i}\right)=1$ and notice that $\left|f_{i}\left(x_{i}-x_{j}\right)\right| \leqslant\left\|x_{i}-x_{j}\right\| \leqslant 1, i, j \in \mathbb{N}_{1}$. Hence $f_{i}\left(x_{j}\right) \geqslant 0$ for all $i, j \in \mathbb{N}_{1}$. It is sufficient to put

$$
F(x)=\sum_{n=1}^{\infty} \frac{1}{2^{n}} f_{n}(x)
$$

The general case of $A$ follows easily from the separability of $X$.

(ii) Fix $x \in A$ and consider a supporting functional $F \in S_{X}$. with $F(x)=1$. If $F(y)=0$ for some $y \in A$ we would have $y \in B(x, 1)$ which contradicts the strict convexity of $X$.

The proof of (iii) is similar.

REMARK 4.2. It is not difficult to see that in the case (iii) we can strengthen our result: if $\varepsilon_{0}(X)<1$, then there exists $c>0$ such that for every $A \subset S_{X}$ with $\operatorname{diam} A \leqslant 1$ there exists $F \in X^{*}$ with $F(x) \geqslant c$ for all $x \in A$.

Unfortunately, neither separable, nor strictly convex Banach spaces are not stable under passing to Banach ultrapowers. On the other hand, $\varepsilon_{0}(X)=\varepsilon_{0}\left((X)_{U}\right)$ and we obtain another proof of the well known result that spaces with $\varepsilon_{0}(X)<1$ have FPP.

The first example of a Banach space without property $(S)$ is due to $\mathrm{K}$. Bolibok. He observed (private communication) that $l_{\infty}\left(\aleph_{1}\right)$ does not have property $(S)$. The following example shows that even a superreflexive space need not possess the $(S)$ property.

EXAMPLE 4.3. Let $X_{\sqrt{2}}(\Gamma)$ be the space $l_{2}(\Gamma)$ with the following equivalent norm:

$$
\|x\|=\max \left\{\|x\|_{2}, \sqrt{2}\|x\|_{\infty}\right\},
$$

where $x \in l_{2}(\Gamma)$. Let $\Gamma$ be an uncountable set and take $A=\left\{(1 / \sqrt{2}) e_{\alpha}: \alpha \in \Gamma\right\}$, where $\left\{e_{\alpha}: \alpha \in \Gamma\right\}$ is an orthonormal basis for $l_{2}(\Gamma)$. It is not difficult to see that $A \subset S_{X}$ and 
that $\operatorname{diam} A=1$. But clearly there is no continuous linear functional which is strictly positive on $A$. Note that $X_{\sqrt{2}}(\Gamma)$ is $\sqrt{2}$-isomorphic to a non-separable Hilbert space.

Though $X_{\sqrt{2}}(\Gamma)$ does not have property $(S)$, it will follow from Theorem 4.7 that it has, however, property $\left(S_{m}\right)$.

Let us recall that a Banach space $X$ is uniformly noncreasy (UNC in short) provided that for every $\varepsilon>0$ there is $\delta>0$ such that if $x^{*}, y^{*} \in S_{X}$. and $\left\|x^{*}-y^{*}\right\| \geqslant \varepsilon$, then $\operatorname{diam} S\left(x^{*}, y^{*}, \delta\right) \leqslant \varepsilon$. Here

$$
S\left(x^{*}, y^{*}, \delta\right)=\left\{x \in B_{X}: x^{*}(x) \geqslant 1-\delta \wedge y^{*}(x) \geqslant 1-\delta\right\}
$$

and $\operatorname{diam} \emptyset=-\infty$.

The following facts are known ([19]):

(i) $X$ is UNC if and only if $X^{*}$ is UNC,

(ii) $X$ is UNC if and only if $(X)_{U}$ is UNC,

(iii) if $X$ is UNC, then $X$ is superreflexive,

(iv) all uniformly convex spaces are UNC,

(v) all uniformly smooth spaces are UNC.

The paper [19] provides us with some other examples of uniformly noncreasy spaces. We mention a few.

The Bynum space $l_{2,1}$ (see [5]) is the space $l_{2}$ endowed with the equivalent norm given by the formula

$$
\|x\|_{2,1}=\left\|x^{+}\right\|_{2}+\left\|x^{-}\right\|_{2}
$$

where $x \in l_{2}, x^{+}, x^{-}$denote the positive and negative parts of $x$, respectively. Similarly, $l_{2, \infty}$ is the space $l_{2}$ with the norm

$$
\|x\|_{2, \infty}=\max \left\{\left\|x^{+}\right\|_{2},\left\|x^{-}\right\|_{2}\right\} .
$$

It is proved in [19] that the Bynum spaces are UNC. It is also proved there that $X_{\sqrt{2}}(\mathbb{N})$ is UNC and an easy generalisation gives that $X_{\sqrt{2}}(\Gamma)$ is UNC for any set $\Gamma$. Note that both $l_{2, \infty}$ and $X_{\sqrt{2}}(\Gamma)$ do not have normal structure (see $[2,5]$ ).

The following theorem shows that the concept of uniformly noncreasy spaces yields a class of superreflexive spaces with the fixed point property.

TheOREM 4.4. ([19]) If a Banach space $X$ is UNC, then $X$ has FPP.

Unfortunately, there exist "very simple" superreflexive spaces with FPP which are not uniformly noncreasy.

EXAmple 4.5. Consider the space $\mathbb{R}^{3}$ with the norm "maximum". It is easy to see that this is not a uniformly noncreasy space. An easy modification of the norm provides us with the example of a Banach space with $\varepsilon_{0}(X)<1$ which is not UNC.

We shall show that all uniformly noncreasy spaces have property $\left(S_{m}\right)$. 
LEMma 4.6. Let $X$ be a reflexive space and assume that there exists $A \subset S_{X}$ with $\operatorname{diam} A \leqslant 1$ which cannot be separated from zero:

$$
\forall F \in X^{*} \exists x \in A(F(x)=0) .
$$

Then, for every $\varepsilon>0$, there exist $x, y \in A$ and $f, g \in S_{X}$. such that

$$
f(x)=1, g(y)=1,0 \leqslant f(y)<\varepsilon, 0 \leqslant g(X)<\varepsilon .
$$

Proof: Let $A^{\text {wcl }}$ be the weak closure of $A$. For each $x \in A$ take a supporting functional $f_{x} \in S_{X}$. with $f_{x}(x)=1$. Let us notice that $f_{x}(y) \geqslant 0$ for all $x \in A$ and $y \in A^{\mathrm{wcl}}$. Assume first that

$$
A^{\mathrm{wcl}} \subset \bigcup_{x \in A}\left\{y \in X: f_{x}(y)>0\right\} .
$$

Then, from the weak compactness of $A^{\text {wcl }}$, we have

$$
A^{\mathrm{wcl}} \subset \bigcup_{k=1}^{n}\left\{y \in X: f_{x_{k}}(y)>0\right\}
$$

for some $x_{1}, \ldots, x_{n} \in A$. Putting $F(y)=1 / n \sum_{k=1}^{n} f_{x_{k}}(y)$ we obtain $F(y)>0$ for $y \in A^{\text {wcl }}$, a contradiction.

Thus, we may assume that $f_{x}(w)=0$ for some $w \in A^{\text {wcl }}$ and all $x \in A$. Then, there exists a sequence $\left\{x_{n}\right\}$ of points in $A$ tending weakly to $w$. From the weak compactness of $B_{X}$. we may assume that a sequence of supporting functionals $\left\{f_{x_{n}}\right\}$ tends weakly to some $f_{0} \in B_{X}$. and hence $f_{0}(w)=0$. Fix $\varepsilon>0$ and take $n_{0} \in \mathbb{N}$ such that $f_{0}\left(x_{n}\right)<\varepsilon$ for $n \geqslant n_{0}$. Then, there exists $m_{0} \in \mathbb{N}$ such that $f_{x_{m}}\left(x_{n_{0}}\right)<\varepsilon$ for $m \geqslant m_{0}$. Since $\left\{x_{n}\right\}$ tends weakly to $w$ we have also $f_{x_{n_{0}}}\left(x_{m}\right)<f_{0}\left(x_{m}\right)+\varepsilon / 2<\varepsilon$ for sufficiently large $m \geqslant m_{0}$. It is enough to consider the elements $x_{n_{0}}, x_{m}$ and the corresponding functionals.

THEOREM 4.7. If $X$ is UNC, then $X$ has property $\left(S_{m}\right)$.

Proof: We shall follow the ideas from [19] but we have to deal more delicate. Let us assume that there exists a metrically convex set $A \subset S_{X}$ with $\operatorname{diam} A=1$ which cannot be separated from zero. Fix $\varepsilon \in(0,1 / 3)$ and take $\delta \in(0,1 / 3)$ such that

$$
\operatorname{diam} S\left(x^{*}, y^{*}, \delta\right) \leqslant \varepsilon
$$

whenever $x^{*}, y^{*} \in S_{X}$ and $\left\|x^{*}-y^{*}\right\| \geqslant \varepsilon$. Then there exists $\delta_{1} \in(0,1 / 3)$ such that

$$
\operatorname{diam} S\left(x^{*}, y^{*}, \delta_{1}\right) \leqslant \delta
$$

whenever $x^{*}, y^{*} \in S_{X}$ and $\left\|x^{*}-y^{*}\right\| \geqslant \delta$. 
From Lemma 4.6, there exist $x, y \in A$ and $f, g \in S_{X}$. such that $f(x)=1, g(y)=$ $1,0 \leqslant f(y)<\left(\delta_{1} / 2\right), 0 \leqslant g(X)<\delta_{1} / 2$. Since $A$ is metrically convex we have $\|w-x\|=$ $\|w-y\|=\|x-y\| / 2$ for some $w \in A$. Then

$$
\frac{1}{2} \leqslant f(w) \leqslant \frac{1}{2}\|x-y\|+f(y) \leqslant \frac{1}{2}+\frac{\delta_{1}}{2}
$$

and similarly

$$
\frac{1}{2} \leqslant g(w) \leqslant \frac{1}{2}+\frac{\delta_{1}}{2}
$$

Let us notice that $2(x-w), 2(w-y) \in S\left(f,-g, \delta_{1}\right)$. Moreover $\|f+g\| \geqslant(f+g)(x) \geqslant$ $1>\delta$ and we deduce from (4.1) that

$$
\|x+y-2 w\|=\frac{1}{2}\|2(x-w)-2(w-y)\| \leqslant \frac{1}{2} \delta \leqslant \delta .
$$

Put $h \in S_{X^{*}}$ with $h(w)=\|w\|=1$. Then $|h(x+y-2 w)| \leqslant \delta$ and consequently $h(x+y) \geqslant 2-\delta$. Hence $h(x) \geqslant 1-\delta$ and $h(y) \geqslant 1-\delta$. Since $A$ is not separated from zero, there exists $z \in A$ such that $f(z)=0$ and $h(z)=0$ (if not, we put $F=(f+h) / 2$ which separates $A$ from zero). Therefore $x, x-z \in S(f, h, \delta)$ and $\|h-f\| \geqslant(h-f)(y) \geqslant$ $1-\delta-\delta_{1} / 2>1 / 3>\varepsilon$. Consequently $\|z\|=\|x-(x-z)\| \leqslant \varepsilon$ and we obtain a contradiction.

Let ut notice that Theorem 4.4 is now an immediate consequence of Theorems 3.1, 4.7 and the fact that uniformly noncreasy spaces are stable under passing to Banach ultrapowers.

DEFINITION 4.8. A Banach space is said to have the $\left(S^{U}\right)$ property (respectively the $\left(S_{m}^{U}\right)$ property) if there exists a free ultrafilter $U$ defined on $\mathbb{N}$ such that $(X)_{U}$ has property $(S)\left(\left(S_{m}\right)\right.$ respectively).

We can summarise our considerations given above in the following.

PROPOSITION 4.9 .

(i) All finite dimensional Banach spaces have property $\left(S^{U}\right)$.

(ii) All Banach spaces $X$ with $\varepsilon_{0}(X)<1$ have property $\left(S^{U}\right)$.

(iii) All uniformly noncreasy spaces have $\left(S_{m}^{U}\right)$ property.

(iv) If a superreflexive space $X$ has property $\left(S_{m}^{U}\right)$, then $X$ has FPP.

To give further examples of superreflexive spaces with property $\left(S^{U}\right)$, denote by $\left(X_{1} \oplus \ldots \oplus X_{n}\right)_{\infty}$ the product $X_{1} \times \ldots \times X_{n}$ of Banach spaces $X_{1}, \ldots, X_{n}$ with the norm given by the formula $\left\|\left(x_{1}, \ldots, x_{n}\right)\right\|_{\infty}=\max \left\{\left\|x_{1}\right\|, \ldots,\left\|x_{n}\right\|\right\}$. It was proved in [19] that if Banach spaces $X_{1}, X_{2}$ are uniformly convex and uniformly smooth, then the space $\left(X_{1} \oplus X_{2}\right)_{\infty}$ is uniformly noncreasy. Note that it is not the case for $\left(X_{1} \oplus X_{2} \oplus X_{3}\right)_{\infty}$ (see Example 4.5). However we have the following. 
Proposition 4.10. Let $X_{1}, \ldots, X_{n}$ be Banach spaces with $\varepsilon_{0}\left(X_{i}\right)<1, i=$ $1,2, \ldots, n$. Then $\left(X_{1} \oplus \ldots \oplus X_{n}\right)_{\infty}$ has property $\left(S^{U}\right)$.

Proof: Let us first notice that

$$
\left(\left(X_{1} \oplus \ldots \oplus X_{n}\right)_{\infty}\right)_{U}=\left(\left(X_{1}\right)_{U} \oplus \ldots \oplus\left(X_{n}\right)_{U}\right)_{\infty}
$$

and therefore it is sufficient to prove that $\left(X_{1} \oplus \ldots \oplus X_{n}\right)_{\infty}$ has the $(S)$ property. Let $A \subset S_{X}, \operatorname{diam} A=1$. Denote by $P_{i}, i=1, \ldots, n$, the projections $P_{i}\left(x_{1}, \ldots, x_{n}\right)=x_{i}$ and put $A_{i}=\left\{x=\left(x_{1}, \ldots, x_{n}\right) \in A:\left\|P_{i} x\right\|=1\right\}$. Let $I=\left\{i \in\{1, \ldots, n\}: A_{i} \neq \emptyset\right\}$. Obviously $A=\bigcup_{i \in I} A_{i}$. For each $A_{i}, i \in I$, fix $y^{i}=\left(y_{1}^{i}, \ldots, y_{n}^{i}\right) \in A_{i}$ and take $f_{i} \in S_{X_{i}}$ with $f_{i}\left(y_{i}^{i}\right)=1$. Then

$$
\left|f_{i}\left(x_{i}\right)-f_{i}\left(y_{i}^{i}\right)\right| \leqslant\left\|x_{i}-y_{i}^{i}\right\| \leqslant\left\|x-y^{i}\right\|_{\infty} \leqslant 1
$$

for every $x=\left(x_{1}, \ldots, x_{n}\right) \in A$ and hence $f_{i}\left(x_{i}\right) \geqslant 0, i \in I, x \in A$. Moreover $f_{i}\left(x_{i}\right)>0$ for $x \in A_{i}$. It is enough to put $F\left(x_{1}, \ldots, x_{n}\right)=\sum_{i \in I} f_{i}\left(x_{i}\right)$.

REMARK 4.11. In Sections 3 and 4 we only consider free ultrafilters defined on the set natural numbers $\mathbb{N}$. But we can extend our results without much effort for arbitrary countably incomplete ultrafilters defined on a certain set $I$ (see also [21]).

\section{REFERENCES}

[1] A.G. Aksoy and M.A. Khamsi, Nonstandard methods in fixed point theory, Universitext (Springer-Verlag, Berlin, Heidelberg, New York, 1990).

[2] L.P. Belluce, W.A. Kirk and E.F. Steiner, 'Normal structure in Banach spaces', Pacific J. Math. 26 (1968), 433-440.

[3] F.E. Browder, 'Fixed point theorems for noncompact mappings in Hilbert space', Proc. Nat. Acad. Sci. U.S.A. 43 (1965), 1272-1276.

[4] F.E. Browder, 'Nonexpansive nonlinear operators in a Banach space', Proc. Nat. Acad. Sci. U.S.A. 54 (1965), 1041-1044.

[5] W.L. Bynum, 'A class of Banach spaces lacking normal structure', Compositio Math. 25 (1972), 233-236.

[6] P.N. Dowling and C.J. Lennard 'Every nonreflexive subspace of $L_{1}[0,1]$ fails the fixed point property', Proc. Amer. Math. Soc. 125 (1997), 443-446.

[7] J. Elton, P.K. Lin, E. Odell and S. Szarek, 'Remarks on the fixed point problem for nonexpansive maps', Contemp. Math. 18 (1983), 87-120.

[8] P. Enflo, 'Banach spaces which can be given an equivalent uniformly convex norm', Israel J. Math. 13 (1972), 281-288.

[9] J. Garcia Falset, 'The fixed point property in Banach spaces with NUS-property', $J$. Math. Anal. Appl. 215 (1997), 532-542.

[10] K. Goebel and W.A. Kirk, Topics in metric fixed point theory (Cambridge University Press, Cambridge, 1990).

[11] D. Göhde, 'Zum Prinzip der kontraktiven abbildung', Math. Nachr. 30 (1965), 251-258. 
[12] S. Heinrich, 'Ultraproducts in Banach space theory', J. Reine Angew. Math. 313 (1980), 72-104.

[13] C.W. Henson and L.C. Moore, Jr., 'The nonstandard theory of topological vector spaces', Trans. Amer. Math. Soc. 172 (1972), 405-435.

[14] C.W. Henson and L.C. Moore, Jr., 'Subspaces of the nonstandard hull of a normed space', Trans. Amer. Math. Soc. 197 (1974), 131-143.

[15] R.C. James, 'Super-reflexive Banach spaces', Canad. J. Math. 24 (1972), 896-904.

[16] W.A. Kirk, 'A fixed point theorem for mappings which do not increase distances', Amer. Math. Monthly 72 (1965), 1004-1006.

[17] P.K. Lin, 'Unconditional basis and fixed points of nonexpansive mappings', Pacific J. Math. 116 (1985), 69-76.

[18] B. Maurey, Points fixes des contractions de certains faiblement compact de $L^{1}$, Seminaire d'Analyse Fonctionelle 1980-81 (Ecole Polytechnique, Palaiseau, 1981).

[19] S. Prus, 'Banach spaces which are uniformly noncreasy', Proc. 2nd World Congress of Nonlinear Analysts (Athens, 1996), Nonlin. Anal. 30 (1997), 2317-2324.

[20] B. Sims, Ultra-techniques in Banach space theory, Queen's Papers in Pure and Applied Math. 60 (Queens University, Ontario, 1982).

[21] A. Wiśnicki, 'Neocompact sets and the fixed point property', J. Math. Anal. Appl. (to appear).

Department of Mathematics

Maria Curie -Skłodowska University

20-031 Lublin

Poland

e-mail: awisnic@golem.umcs.lublin.pl 